\title{
A Dynamic Model of Segregation in Small-World Networks
}

\author{
Giorgio Fagiolo, Marco Valente, and Nicolaas J. Vriend
}

\begin{abstract}
Schelling [19-22] considered a simple model with individual agents who only care about the types of people living in their own local neighborhood. The spatial structure was represented by a one- or two-dimensional lattice. Schelling showed that an integrated society will generally unravel into a rather segregated one even though no individual agent strictly prefers this. We make some steps to generalize the spatial proximity model to a proximity model of segregation. That is, we examine models with individual agents who interact "locally" in a range of network structures with topological properties that are different from those of regular lattices. Assuming mild preferences about with whom they interact, we study best-response dynamics in random and regular non-directed graphs as well as in small-world and scale-free networks. Our main result is that the system attains levels of segregation that are in line with those reached in the lattice-based spatial proximity model. That is, Schelling's original results seem to be robust to the structural properties of the network. In other words, mild proximity preferences coupled with adjustment dynamics can explain segregation not just in regular spatial networks but also in more general social networks.
\end{abstract}

G. Fagiolo

Laboratory of Economics and Management, Sant'Anna School of Advanced Studies, Pisa, Italy giorgio.fagiolo@sssup.it

M. Valente

Faculty of Economics, University of L'Aquila, L'Aquila, Italy

mv@business.auc.dk

N.J. Vriend

Department of Economics, Queen Mary, University of London, London, UK

n.vriend@qmul.ac.uk

A.K. Naimzada et al. (eds.), Networks, Topology and Dynamics, 


\section{Introduction}

Segregation has been widely recognized as a critical issue, from both a sociopolitical and a public-economic perspective, in many Western countries. In the USA, segregation has dominated the political debate for some time in the second half of the last century. More recently, segregation issues are increasingly becoming one of the main points in the political agenda of the majority of European countries, and this trend is likely to be reinforced by the geo-political turmoils due to the events following 9/11 and the ongoing enlargement process of the EU.

The main problem faced by countries trying to reduce segregation is that we still do not know how to attain this goal. Indeed, the plethora of integration policies that have been implemented in the last decades turned out to be almost completely ineffective. In particular, all policies aimed at changing individual preferences towards multiculturalism (e.g., by promoting people openness and tolerance with respect to diversity) did not substantially improve integration [3]. Therefore, gaining a better knowledge of the forces underlying the dynamics leading to segregated societies seems crucial today as it was in the second half of the 20th century.

Exactly in those years, Schelling [19-22] studied a simple model of segregation with individual agents who only care about the types of people living in their own local neighborhood. The spatial structure was represented by a one- or twodimensional lattice. Schelling showed that an integrated society will generally unravel into a rather segregated one even though no individual agent strictly prefers this. Rather, segregation seemed due to the spontaneous dynamics of the economic forces, with all individuals following their incentives to move in the most attractive locations. The preferences considered in the spatial proximity model are said to be mild, as everybody would be happy in a perfectly integrated society.

More recently, Pancs and Vriend [17] examined the robustness of Schelling's spatial proximity model. They showed that the model can be further simplified (rendering the individual preferences even more salient as an explanatory variable of segregation), and that these proximity preferences may be even more extreme in favor of integration. This focus on mild individual preferences or preferences that even favor integration is not to say that institutional constraints or racism may not hinder integration. But what the model shows is that even without such obstacles one should perhaps expect segregation. It seems that any integration policy must be based on a good understanding of these spontaneous dynamics.

Both original Schelling's model and Pancs and Vriend's robustness analyses explore segregation dynamics on regular (one- or two-dimensional) lattices. In other words, they both study the emergence of segregation in a geographical space. Indeed, lattices are widely employed in local-interaction models because they can be considered as a first approximation of geographical space [8]. The idea that people care about their spatial proximity can be justified by the fact that this is where people mow their lawn, where their children play outside, where they do their shopping, and where they park their car. The social environment is, however, not limited to this spatial proximity. People also interact through networks of friends, relatives, 
and colleagues, and through virtual communities on the internet. And they are likely to have preferences with whom they do this, just as they have preferences about their spatial proximity.

This suggests that segregation need not necessarily occur at the spatial (neighborhood) level: one might conceive people who are socially segregated despite being spatially integrated. This appeared to be the case with some of the recent terror suspects in the Netherlands and the UK. Therefore, a better understanding of the phenomenon of segregation in more general network structures seems desirable.

In this paper, we generalize Schelling's spatial proximity model to a proximity model of segregation where individual agents interact "locally" in a range of social network structures with topological properties that are different from those of regular lattices. Among all network structures alternative to regular lattices, we explore in particular small-world networks, which have been found to be a good proxy of real-world social interaction structures [2]. We stick to standard assumptions as far as types and preferences are concerned, and we study the ensuing best-response dynamics in two setups. In the first one (global-move setup) agents that are not satisfied with their current state can choose uniformly at random any empty location in the whole network (i.e., move arbitrarily far away in the social space). In the second setup (local-move), they are bound to choose one of the available locations in their social neighborhood (if any).

The rest of the paper is organized as follows. Section 2 discusses in more detail the classes of networks that we consider in our analysis. In Sects. 3 and 4 we present the model in its global- and local-move variants, and we discuss its implementation. Section 5 introduces the index that we employ to measure segregation in social networks. Simulation results are in Sect. 6, which also contains a sensitivity analysis of the parameter space. Finally, Sect. 7 concludes.

\section{Social Networks and Small Worlds}

The last fifteen years have witnessed an incredible outburst of empirical studies on natural, social and economic networks [1, 16, 23, 24]. More specifically, the bulk of contributions has focused on the structural and topological properties of empiricallyobservable networks such as the Internet and the WWW, airline connections, scientific collaborations and citations, trade and labor-market contacts, friendship and other social relationships, business relations and R\&D partnerships, cellular, ecological and neural networks.

The main message of this vast literature is that most real-world networks belong to a particular class of structures that display neither the intrinsic spatial regularity of lattices, nor the disorder of random graphs [i.e., networks where any two agents are neighbors, independently of all the others, with some given probability, see 5]. To see why, let us begin with some basic definitions.

It is well-known that the simplest mathematical description of a network can be given in terms of an undirected graph $G=(n, A)$, where $n$ is the number of nodes 
(individuals) and $A$ is a $n \times n$ symmetric matrix whose generic element $a_{i j}$ is equal to 1 if nodes $i$ and $j$ are linked by an edge (i.e., they are neighbors, either in a geographical or a social space), and 0 otherwise. Different networks can be taxonomized according to their structural and topological properties [18, 23, 24]. The most salient characteristics of a network can be summarized by the distributions of three statistics: (1) degree of a node; (2) clustering of a node; (3) shortest-path length between any two nodes. The degree of a node is simply the number of neighbors it has. Lattices are regular graphs because all nodes have the same degree. In random graphs node degrees are heterogeneous and symmetrically distributed around the average degree, which is proportional to the probability that any two nodes are neighbors. The clustering of a node is instead the likelihood that any two neighbors of that node are themselves neighbors. For each node $i$, this can be easily computed by counting the number of triangles with $i$ as one vertex (and dividing this number by the total number of triangles that $i$ could have formed given its degree). Obviously, lattices are in general much more clustered than random graphs, as their nodes are typically distributed in tightly connected clusters where any two neighbors are also neighbors by construction. ${ }^{1}$ Finally, the shortest path length between any two nodes $(i, j)$ is defined as the minimum number of links that one has to traverse to get from $i$ to $j$. This measure has been popularized as the "degrees of separation," see Watts [26]. Again, lattices are extreme cases where this measure is generally high, as any two nodes far away in the lattice can reach each other only by travelling through all nodes that are in between. More precisely, the average distance between any two edges increases as $\sqrt{N}$, much faster than in random graphs, where it only increases as $\ln N$.

Recent empirical studies [see 1, 16] have shown that real-world social networks are neither regular lattices nor random graphs, but lie in between. Indeed, they belong to the class of "small worlds" $[13,15,25]$. This type of networks preserves the high clustering level displayed by lattices, while exhibiting a smaller average shortest-path length, which only increases as $\ln N$ as in random graphs. This means that individuals embedded in real-world social networks tend to form tightly connected local clusters (of friends, relatives, business partners, etc.) as happens in geographical space. However, these local clusters are also frequently connected among them by shortcuts that allow any two agents who are arbitrarily far away in the social space to reach each other in a few steps [actually only six, on average, in many cases; see 15, for a review].

Networks belonging to the small-world class strongly differ, however, as to the shape of their degree distributions [2]. A first sub-class, which we will label as "Watts-Strogatz" (WS) in what follows, exhibits a quasi-symmetric degree distribution, centered around the average $d>0$ (and tails possibly decaying exponentially fast as in the Gaussian distribution). To the second type of small-world networks belong the so-called "scale-free" networks (henceforth SF), i.e., networks whose degree distribution is right-skewed and decays with a power-law tail. Therefore, in WS small-world networks most of the nodes have the same degree. On the contrary,

1 This may not be the case, however, for some particular choices of the metrics (e.g., the Von-Neumann one) and a relatively small interaction radius (e.g., equal to one). More on that below. 

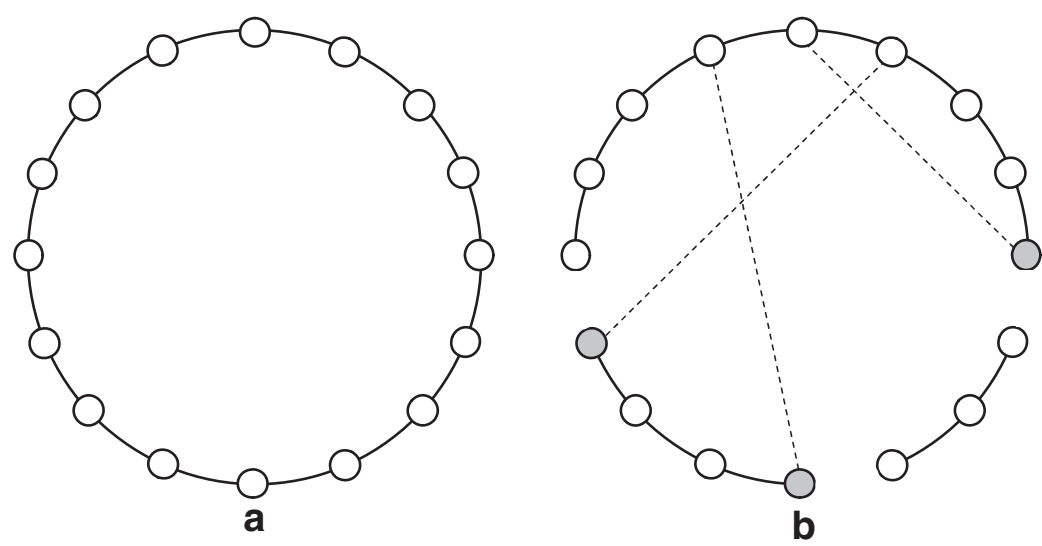

Fig. 1 Rewiring procedure for WS small-world graphs. Panel (a): we start from a circle where each node is connected with two neighbors. Panel (b): the graph after three nodes (in grey) have successfully rewired one of their links. Dashed lines depict rewired links

Fig. 2 An example of a WS graph obtained from $M=100$ nodes originally lying on a circle and interacting with two neighbors only $(r=1)$. Rewiring probability $\beta=0.2$. Node colors depend on degree after rewiring. Black nodes: $d \geq 3$. Grey nodes: $d=2$. White nodes: $d=1$

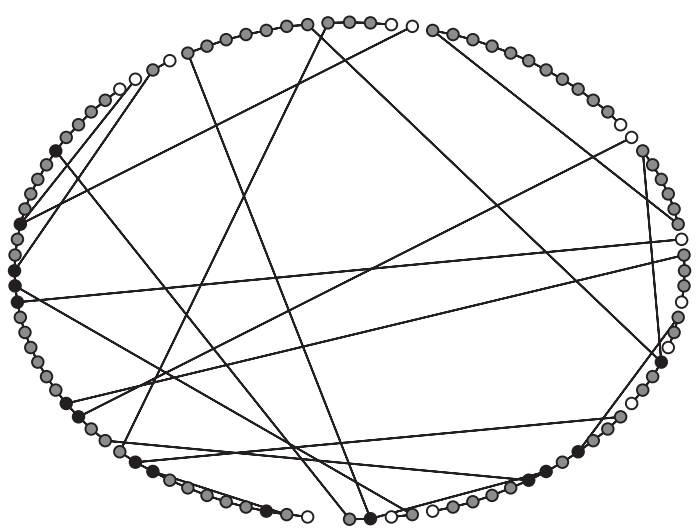

SF networks are characterized by a few nodes holding many partners (i.e., the hubs) and many nodes holding a few partners.

WS and SF small-world networks also differ in their generating mechanism [6]. Think of a given graph $G$ as the (long-run) equilibrium of some stochastic dynamic model of node and edge dynamics. The most simple way to generate WS graphs runs as follows [27]. Start with a regular lattice (e.g., a circle), where each node has two neighbors - one on the left, one on the right, as in Fig. 1, panel (a). At each $t=0$, pick a node (say $i$ ) at random and rewire with probability $\beta \in[0,1]$ one of its two links (say the one with $j$ ) to another node in the graph (say $h \neq i$ ) chosen at random. Figure 1, panel (b) shows the graph after three successful rewirings (nodes that have successfully rewired are shown in grey). If one repeats this procedure for a large number of times (avoiding the case that more than one link connects the same couple of nodes), the resulting graph is a small world, provided that $\beta$ is sufficiently small (typically between 0.01 and 0.3 ), see Fig. 2 for an example. Notice how a small fraction of nodes (in black) hold more than two links, with some of their links being to nodes located arbitrarily far from them on the circle, while the other nodes (in grey or white) who kept only one or two links are usually linked to their direct neighbors only. This mechanism allows one to span the space of a wide variety of networks, from lattices $(\beta=0)$ to random graphs $(\beta=1)$. 
Fig. 3 An example of a SF graph obtained applying a preferential attachment algorithm. The initial population size is $M_{0}=16$. All initial nodes have degree $d=4$. Final population size is $M=100$. We only plot a subset of all nodes for simplicity. White nodes are the "hubs"

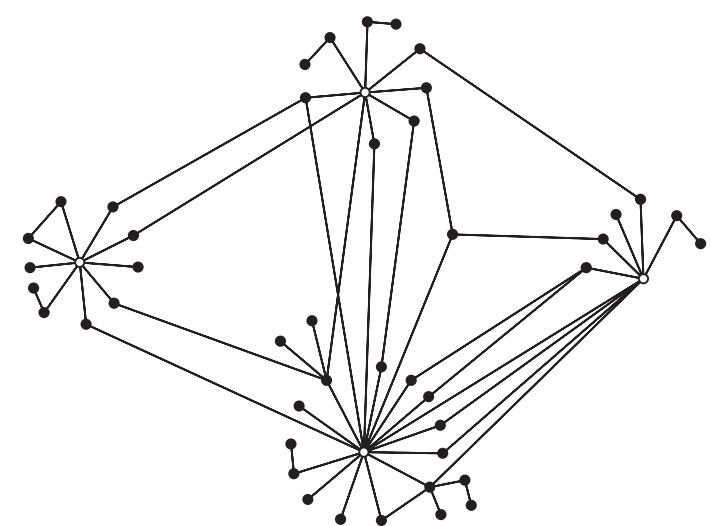

SF networks can instead be generated as the limit of a growth process known as "preferential attachment" [4]. According to this algorithm, one starts from an initial graph $G_{0}$ (e.g., a small lattice) and adds a new node to the graph in each step. The newly added node makes new connections with existing nodes, where the probability of connecting with any existing node is proportional to the current degree of the latter. As this process goes on, better-connected nodes attract more and more entrants (i.e., the rich get richer). The resulting (limit) graph can be shown to be a small world with a power-law degree distribution. The underlying assumption of this setup is that any node can hold at no cost any arbitrarily large number of nodes (as network size increases). As Fig. 3 shows for an instance of a SF graph obtained by applying the above procedure for $n=100$, a few "hubs" (in white) holding a large number of links coexist with many nodes (in black) connected with a small number of other nodes, and possibly with the hubs.

To sum up: Recent empirical works have robustly highlighted that small-world (WS and SF) networks ubiquitously emerge in many social contexts. Therefore, small-world networks seem to be the most natural candidate to test the robustness of Schelling's spatial proximity model when agents are placed in more general types of networks. In the following section we shall present an extension of the basic Schelling's model that explores this direction.

\section{The Model}

Consider a society composed of $N$ agents who can locate themselves in one of the $M \geq N \geq 3$ available locations. Each location can contain at most one agent. Locations can be connected or not. We model locations and connections through a graph $G$ composed of $M$ nodes and a collection of non-directed edges linking any pair of nodes. Edges are described by the (symmetric) $M \times M$ matrix $W=\left\{w_{k h}\right\}$, where $w_{k k}=0 \forall k=1, \ldots, M$ and $w_{k h}=w_{h k}=1$ if and only if there is an edge connecting nodes $k$ and $h$, and zero otherwise. We define the "neighborhood" $V_{k}$ (or the "interaction group") of a node $k$ as the set of nodes that node $k$ is linked to

$$
V_{k}=\left\{h \in I_{M}: w_{k h}=w_{h k}=1\right\},
$$

where $I_{M}=\{1, \ldots, M\}$. 
We suppose that each node is empty (i.e., it does not contain an agent) with probability $\theta \in(0,1)$, while it is occupied with probability $1-\theta$. Therefore, on average, there are $N=(1-\theta) M$ agents in the society. Each agent can be one of two types, say -1 and +1 . Time is discrete, and time ticks are labeled by $t=0,1,2, \ldots$.

Agents have standard, binary, Schelling-type preferences: they are happy if and only if the relative frequency of agents of their own type is greater or equal than 0.50 in their neighborhood. More formally, if node $i$ is occupied by an agent of type $s \in\{-1,+1\}$ at time $t$

$$
u_{i t}=u_{i t}(s)= \begin{cases}1, & \text { if } x_{i t}(s) \geq 0.5 \\ 0, & \text { otherwise }\end{cases}
$$

where $u_{i t}=u_{i t}(s)$ is the utility of agent $i$ (of type $s$ ) at time $t$ and $x_{i t}(s)$ is the current relative frequency of agents (i.e., filled nodes) of type $s$ in $V_{i \cdot}{ }^{2}$

The initial state of the system is characterized by: (1) an instance of the network structure, i.e., a graph $G_{0}=\left\{I_{M}, W_{0}\right\}$ (more on that below); (2) an allocation of agents and types across the $M$ available nodes. The initial allocation of agents and types across the $M$ nodes is drawn uniform randomly. Thus, at $t=0$, each node $i \in I_{M}$ will be either empty or occupied. If it is occupied, this will be either a -1 or $\mathrm{a}+1$ agent, each with probability 0.5 . Thus, in the society there will be, on average, $N / 2$ agents of type -1 and $N / 2$ agents of type +1 .

The dynamics runs as follows. At each $t>0$, an agent, say $k$, is drawn at random (and independently) from $I_{N}=\{1, \ldots, N\}$. As far as the behavior of the chosen agent is concerned, we shall explore two models:

- Global-Move (GM) Model: Agent $k$ computes the utility that he could earn at each available (i.e., empty) node in the whole network $G_{0}$ (including in the list his current node).

- Local-Move (LM) Model: Agent $k$ computes the utility that he could earn at each available (i.e., empty) node in his neighborhood $V_{k}$ only (including in the list his current node).

Then, in both LM and GM models, agent $k$ chooses the node that provides the highest achievable utility level (i.e., either one of the empty nodes or his current location). Ties are resolved by randomizing among all nodes providing the same maximal utility level. Notice that we assume no inertia in the agents' choices. That is, agents' current locations do not bias their choices (e.g., because of moving costs). The GM model also assumes that agents can move to any empty node in the network, i.e., there are no information or moving constraints or costs (see Sect. 7 for a discussion). Hence, the LM model can be justified by the presence of either a moving cost or some information costs preventing agents to observe anything that is outside their current neighborhood.

\footnotetext{
${ }^{2}$ In line with Pancs and Vriend [17], we assume that the utility associated to an empty neighborhood is zero.
} 


\section{Implementation}

The initial network $G_{0}$ is chosen at random to belong to the small-world class. However, to benchmark our analysis against Schelling's one and Pancs and Vriend's results, we also study the behavior of the model in the case where initial graphs are two-dimensional lattices. More precisely, we experiment with Von-Neumann (VN) and Moore (M) two-dimensional, boundary-less lattices (i.e., torii). It is well-known that neighborhoods $V_{k}$ in two-dimensional lattices are completely defined up to the choice of a metric (specifying how to compute the distance between any two nodes) and an interaction radius $r$. Let $\left(x_{h}, y_{h}\right)$ the coordinates of node $h$ in the lattice. In the $\mathrm{VN}$ case the distance between nodes $\left(k, k^{\prime}\right)$ is given by

$$
\delta_{V N}\left(k, k^{\prime}\right)=\left|x_{k}-x_{k^{\prime}}\right|+\left|y_{k}-y_{k^{\prime}}\right|
$$

while in the Moore case it is equal to

$$
\delta_{M}\left(k, k^{\prime}\right)=\max \left\{\left|x_{k}-x_{k^{\prime}}\right|,\left|y_{k}-y_{k^{\prime}}\right|\right\}
$$

Therefore, if one defines

$$
V_{k}(r)=\left\{h=1, \ldots, M: \delta_{\bullet}(k, h) \leq r\right\}
$$

it is easy to see that in VN lattices all nodes have a degree $d_{V N}=2 r(r+1)$, while in Moore lattices one has $d_{M}=4 r(r+1)$, see Fig. 4 for an illustration.

Initial small-world networks are instead generated using the following procedures:

- WS Graphs: We start from a two-dimensional boundary-less lattice with VN neighborhoods for a certain $r \geq 1$. Then, each edge $(h, k)$ is independently rewired to a randomly chosen node, say $k^{\prime}$, outside $V_{h}(r)$ with some probability $\beta \in(0,1)$. In case of rewiring, the edge $(h, k)$ is deleted and replaced by the

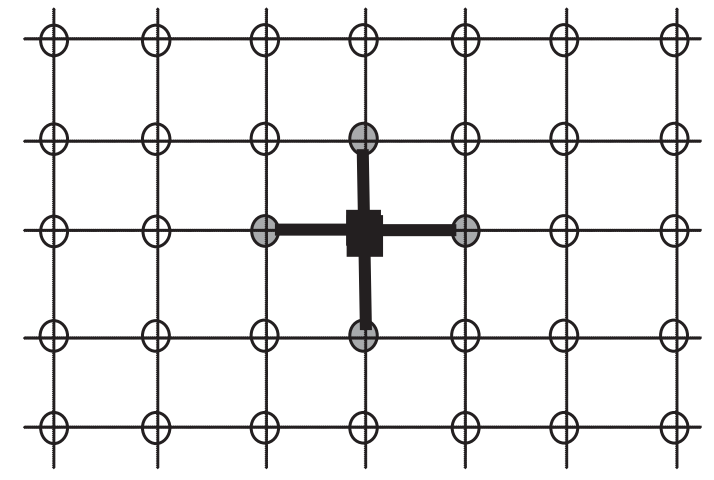

a $2 \mathrm{D}-\mathrm{VN}: \quad r=1$

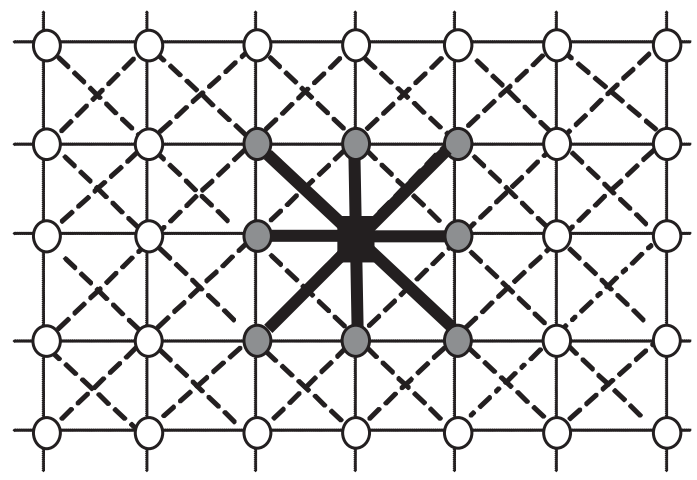

b $2 \mathrm{D}-\mathrm{M}: \quad r=1$

Fig. 4 An example of neighborhood shapes with two-dimensional Von-Neumann (2D-VN) and Moore (2D-M) lattices for $r=1$ 
new edge $\left(h, k^{\prime}\right)$. This yields a symmetric degree distribution, centered around $2 r(r+1)$. In the benchmark results presented below, we employ $\beta=0.2$ and then we study what happens when $\beta$ is tuned in the unit interval.

- SF Graphs: We employ a standard "preferential attachment" procedure, starting with $M_{0}$ nodes linked through a 2D-VN lattice with $r=1$ (and thus an initial degree $d=4$ ). One node at a time is added until a size $M$ is reached. In any step, the additional node is allowed to form 4 links. Each new link is formed by choosing one of the existing nodes with a probability proportional to its current degree.

The model contains a small number of free system- and network-specific parameters. System parameters are $M$ (number of nodes) and $\theta$ (average percentage of empty nodes). Network specific parameters characterize - given the class of networks to be implemented - the set of possible networks from which the one actually in place will be drawn. VN and Moore lattices are characterized by their degree. WS graphs are parameterized by $\beta$ and the degree $d$ of the underlying lattice (before rewiring). Finally, SF graphs depend on the initial population size $M_{0}$. Simulations reveal that average degree $d$ and $M_{0}$ are linked by the following (approximate) relation:

$$
d \simeq 0.00003 \cdot M_{0}^{3}-0.0062 \cdot M_{0}^{2}+0.3485 \cdot M_{0}+3.1916
$$

Notice that $d$ grows for $M_{0} \leq 39$ and decreases for $M_{0} \geq 40$. Hence, in both lattices and small-world graphs, the only common network-specific parameter to be considered is the (average) node degree $d$. WS graphs can also be studied for different $\beta$ levels.

In the lattice-case, the initial graph is automatically defined once one specifies the degree $d$. In small worlds, given a choice for the network class and for the networkspecific parameters of that class (e.g., $d$ and $\beta$ for a WS graph), each time we draw $G_{0}$ uniformly at random from the set of all possible graphs belonging to that class and with the given network-specific parameters.

\section{Measuring Segregation in Networks}

A number of indices have been suggested in the literature to measure segregation when the agents are located on generic networks [see, e.g., 7, 10-12, 14, and references therein]. Here, we will employ Freeman's segregation index (FSI) [11, 12]. The rationale underlying the computation of the FSI is that if a given agent-attribute (in our case the type +1 or -1 ) does not matter for social relationships (i.e., for the link structure as described by $G_{0}$ ), then the links among the agents should be distributed randomly with respect to that attribute. Therefore, suppose we observe a given allocation of agent types across the $M$ nodes, connected through the network $G_{0}$. Let us, then, split the agents in two groups according to their type and, for each type, let us count the number of cross-group links (i.e., the number of links connecting any pair of agents of different types), as well the number of within-group 
links (i.e., the number of links connecting any pair of agents of the same type). This gives us a $2 \times 2$ contingency table whose generic entry $l_{x y}$ gives us the number of links between type- $x$ and type- $y$ agents in $G_{0}$. Similarly, one can compute the expected contingency table for a random allocation of agent types on $G_{0}$. The difference between the number of cross-group ties expected by chance and the number of observed ties (divided by expected ones) gives us the FSI. The index ranges between -1 and 1 , with the highest segregation level obtained when there are no cross-group links in place.

We also check our results against a number of alternative segregation indices, such as the "spectral segregation index" [7], those proposed in Fershtman [10] and Freeman [12], and some of the indices originally developed in the lattice-case [see 17], e.g., the average mix deviation index. As we discuss in Sect. 7, our main results are not qualitatively altered if one considers these alternative segregation measures. Therefore, in what follows we will mainly focus on FSI as our measure of segregation in networks.

\section{Results}

In this section, we explore the behavior of our model for a society of $M=100$ nodes. Our study will take the form of a Monte Carlo (MC) analysis. The procedure is as follows. For each choice of network class and network-specific parameters we generate a number of independent runs. For each run, where necessary, we randomly select a specific instance of the network class, and we generate an initial allocation of agents and types across the network uniformly at random. We, then, let the best-response dynamics run, and collect system statistics when either FSI or the configuration of types across the $M$ nodes have reached a steady-state. This typically happens well before $T=50,000$ time-steps with probability one. We independently repeat this exercise 1,000 times, computing the Monte Carlo (MC) average and standard deviation of FSI. Since across-run variability turns out to be very small (across-run standard deviations are of an order of magnitude of $10^{-5}$ ) and MC distributions appear to be symmetric, we report below MC averages of FSI only.

The main questions we initially address are:

Q1 Are segregation levels in WS and SF small-world networks (as measured by FSI) different from those attained in a society where individuals live in lattices?

Q2 Does the answer to Q1 differ when individuals behave according to a GM or a LM model?

Let us begin with a rough comparison of segregation levels. Figures 5 and 6 show average FSI levels for lattices and small-world graphs in both the GM and LM models. Although segregation seems to be slightly larger in lattices than in WS and SF graphs, overall levels in both lattices and small worlds remain quite large according to FSI (which, we recall, records values close to one only in extreme cases). 
Fig. 5 GM Model. Average FSI levels in lattices, WS and SF graphs for average degrees $d=4,8$. Parameters: $M=100, \theta=0.3$. Note: $d=4$ : VN-lattice; $d=8$ : M-lattice

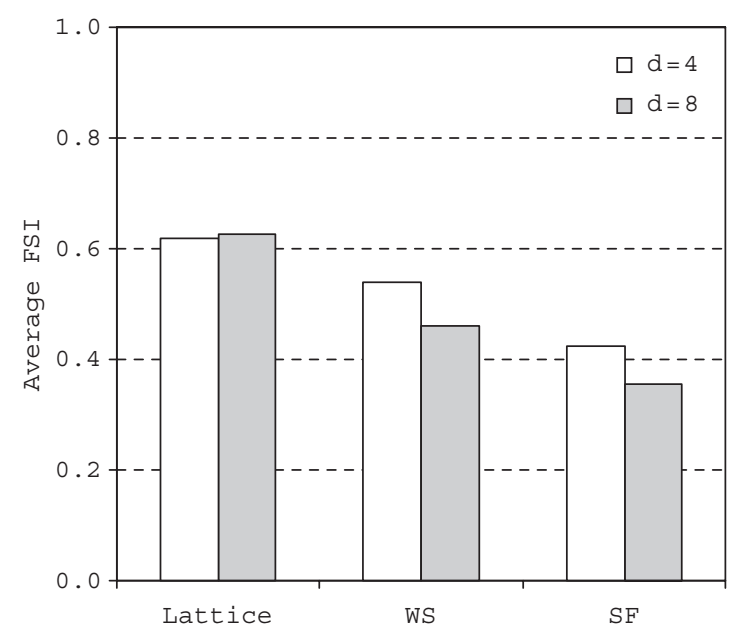

Fig. 6 LM Model. Average FSI levels in lattices, WS and SF graphs for average degrees $d=4,8$. Parameters: $M=100, \theta=0.3$. Note: $d=4$ : VN-lattice; $d=8$ : M-lattice

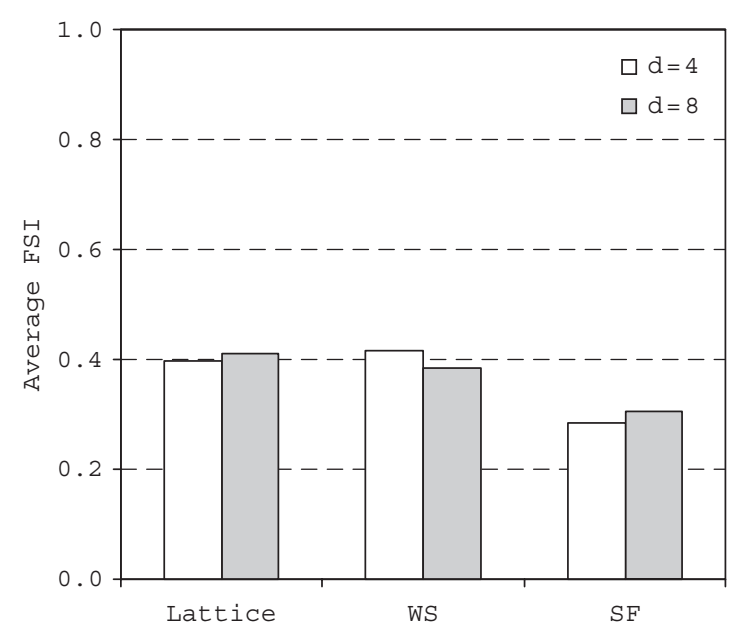

Schelling's results seem to be confirmed when one moves from spatial to social segregation in the GM model.

This result was somewhat expected. When one leaves a lattice world to move in the small-world realm, two important features change. First, the average path length tends to decrease; second, and most important here, neighborhood sizes become heterogeneous (less in WS graphs, more in SF ones). In the GM model agents can freely move in any empty location of the network, no matter their neighborhood size. Hence, it would have been surprising if segregation levels would have substantially changed.

When one comes to the LM model, however, heterogeneity in neighborhood sizes might have some impact on segregation levels. Our simulations instead show that this is not the case, see Fig. 6. Segregation levels in WS and SF networks remain comparable to those in lattices: Schelling's results seem even more robust. Notice also that segregation levels decrease when one moves from a GM to a LM model. In fact, agents in a LM world tend to explore a smaller number of options and the ensuing dynamics turn out to be more "sticky." As a result, high levels of segregation can be attained less easily by the system. 
The above results indicate that both Q1 and Q2 have a simple, common answer: "Not very much." If any, some overall decrease in segregation levels is observed in SF networks. ${ }^{3}$ However, SFI differentials are not so large to draw statisticallysignificant implications (more on that in Sect. 7). ${ }^{4}$

These findings are robust to a sensitivity analysis across system and networkspecific parameters [9]. However, inspection of Figs. 5 and 6 suggests that segregation levels do exhibit some variation with average degree, hinting to some parameter dependence of segregation levels. We can then formulate the following additional questions:

Q3 Do segregation levels in WS and SF small-world networks (as measured by FSI) change with average degree and percentage of empty nodes $(\theta)$ ?

Q4 Do segregation levels in WS networks change with the rewiring parameter $(\beta)$ ?

Let us begin with Q3. Figures 7 and 8 show how segregation levels change with average degree and the percentage of empty nodes in WS and SF graphs for the

Fig. 7 GM Model. Average FSI levels in WS graphs vs. average degree for different levels of $\theta$ and $M=100$

Fig. 8 GM Model. Average FSI levels in SF graphs vs. average degree for different levels of $\theta$ and $M=100$
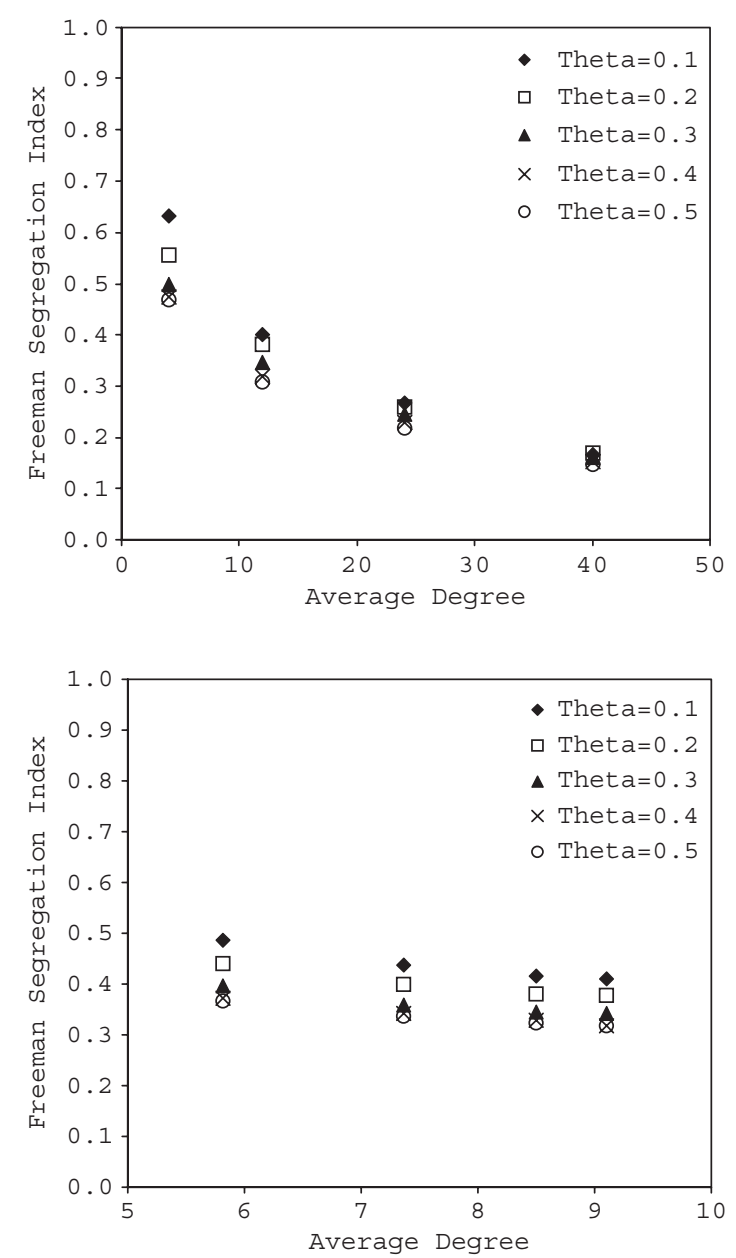

${ }^{3}$ Segregation levels in WS graphs are smaller than in lattices in the GM model only.

${ }^{4}$ Simulations also show that the average values of FSI found throughout our analysis are significantly larger than average FSI values obtained in purely-random allocations of networks and types (when best-response dynamics is not made at work), see Fagiolo et al. [9] for details. 
Fig. 9 LM Model. Average FSI levels in WS graphs vs. average degree for different levels of $\theta$ and $M=100$

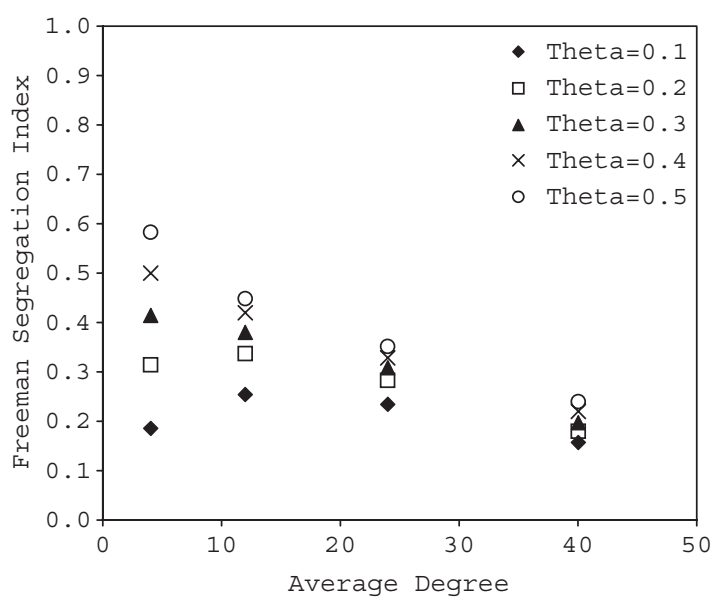

Fig. 10 LM Model. Average FSI levels in SF graphs vs. average degree for different levels of $\theta$ and $M=100$

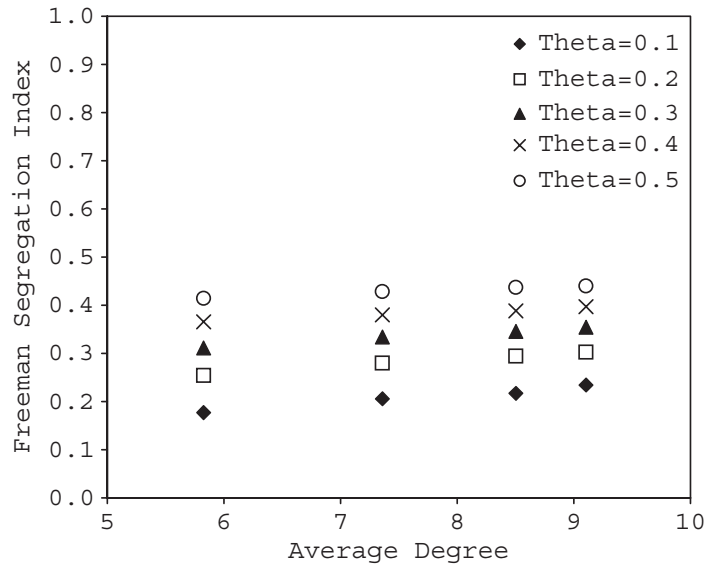

GM model. We see that FSI levels are decreasing with the average degree for any value of $\theta$. Very high segregation levels are attained by the system when the society is poorly connected and there is a small percentage of empty nodes. As the connectivity increases, segregation becomes somewhat less pronounced, but even in very connected societies, segregation levels remain significantly high. Furthermore, segregation tends to decrease in the GM model as the percentage of empty nodes increases, as agents have more degrees of freedom to move around.

The above results substantially change in the LM model, see Figs. 9 and 10. Recall that, in the LM model, degree heterogeneity (which increases as one goes from WS to SF graphs) does now play some role. While more connected WS societies are less segregated, this is not so in SF networks, where segregation mildly increases with average degree. As a result, topological properties of SF networks seem to have an impact on segregating dynamics in our model. Despite more heterogeneity seems to slightly decrease segregation levels given the same connectivity, switching from a less degree-heterogeneous to a more degree-heterogenous society changes the way in which connectivity affects segregation levels.

Finally, Figs. 11 and 12 show segregation levels attained in a WS network for different values of the rewiring probability in both the GM and the LM model. 
Fig. 11 GM Model. Average FSI levels in WS graphs vs. rewiring probability for different average degrees. Parameters: $\theta=0.3$ and $M=100$

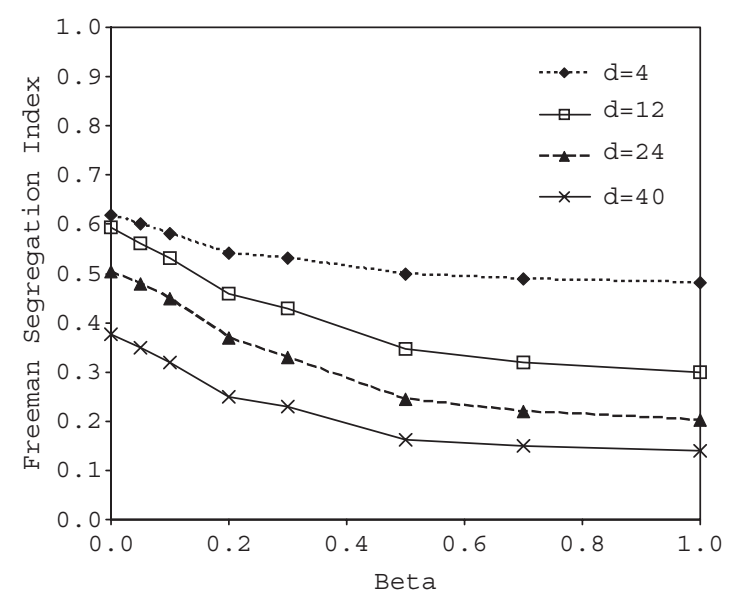

Fig. 12 LM Model. Average FSI levels in WS graphs vs. rewiring probability for different average degrees. Parameters: $\theta=0.3$ and $M=100$

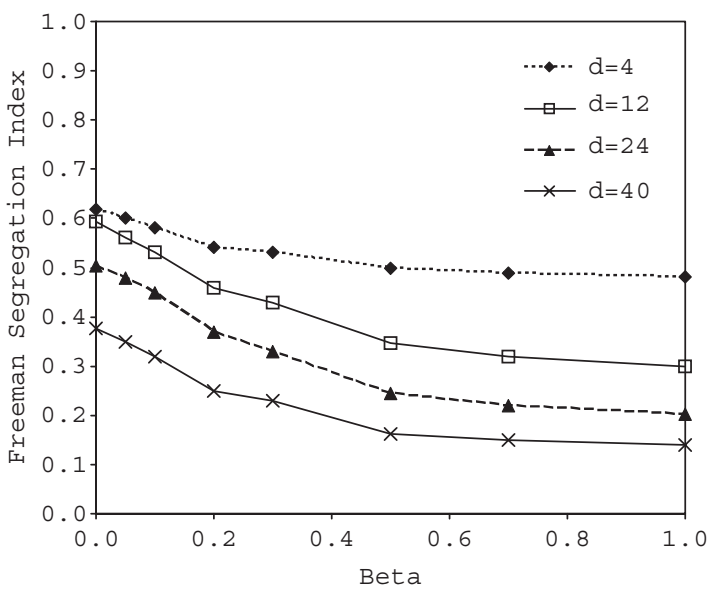

It is easy to see that FSI levels are only mildly decreasing with $\beta$. In the SF case, segregation levels are substantially stable. This implies that, as one interpolates between lattices $(\beta=0)$ and purely random graphs $(\beta=1)$, segregation levels remain quite stable.

\section{Concluding Remarks}

In his seminal contributions, Schelling [19-22] studied a proximity dynamic model of spatial segregation where individuals lived on one- or two-dimensional lattices. He showed that a perfectly integrated society would evolve into a segregated one even though no individual agent would have strictly preferred that outcome in his local neighborhood.

In this paper we have argued that segregation might occur not only in the geographical space, but also in more general social networks. Empirical evidence indicates that in the real-world such networks are neither lattices nor random graphs, but rather belong to the class of small worlds. Building upon this evidence, we have presented a dynamic model of segregation where individuals interact in smallworld social networks. The model sticks to Schelling's original formulation as far 
as individual preferences are concerned and studies best-response dynamics as in Pancs and Vriend [17]. We consider two specifications of the general model, one in which agents can move arbitrarily far away in the social space from their current location (global move model), the other wherein agents can only move in their current social interaction group (local move model).

When one replaces lattices with small worlds, the degree distribution becomes heterogeneous. This heterogeneity is relatively low in Watts-Strogatz (WS) small worlds, while becomes more relevant in scale-free (SF) small worlds. Our main result is that this increase in degree heterogeneity does not dramatically affect Schelling's findings: segregation levels remain comparable to those attained in a lattice world in both a local- or global-move model. We also perform a sensitivity analysis on the parameter space. Simulations show that in the global-move model more connected societies are less segregated. However, when a local-move model is considered, this result does not hold anymore: higher connectivity can imply either smaller or larger segregation levels, depending on the heterogeneity of degrees. The higher heterogeneity, the more likely is that strongly connected societies are also more segregated.

The above results are robust to a series of extensions and modifications [see 9, for details]. These include: (1) segregation measures alternative to the FSI [e.g., the spectral segregation index proposed in 7]; (2) additional network structures such as regular or random graphs; (3) average percentages of empty nodes $(\theta)$ larger than $50 \%$; (4) network size $(M)$.

Furthermore, similar results are obtained if one introduces some "inertia" in the picture. Suppose that an agent located in node $i$ is drawn at random from $I_{N}=\{1, \ldots, N\}$. With inertia, this agent stays put if there is no vacant location that he would strictly prefer to his current location. The idea of inertia is based on the implicit modelling assumption of some small costs of moving (smaller than the smallest possible difference in satisfaction between any two locations, but otherwise arbitrarily small). Notice that under the inertia rule, satisfied agents will never move.

Many interesting issues remain to be explored. First, agents in our model jump from its current location to an available one without being affected by the topology of the network. This implies that average path length has no effect whatsoever in the dynamic process leading to segregation. Therefore, the current formulation of our model does not fully exploit a fundamental difference existing between lattices and small worlds, i.e., the fact that in small worlds average path length tends to decrease. By incorporating into agents' behavioral rules an appropriate algorithm governing the path they follow to travel from their current node to the newly selected one, one might attempt to explore the role played by average path length in the picture.

Finally, our results indicate that degree heterogeneity does affect segregation levels. In particular, when one switches from WS to SF networks, segregation seems to generally decrease and the way in which connectivity affects segregation substantially changes. Therefore, a deeper understanding of the behavior of the model in SF graphs seems desirable. 
Acknowledgements We thank Yannis Ioannides, Giulia Iori, Alan Kirman, Akira Namatame, workshop participants of iNeck (Strasbourg), WEHIA 2005 (Colchester), SCE 2005 (Washington DC) and Complexity 2006 (Aix en Provence) as well as the theory reading group at QM (London) for their comments. Part of this work was done while G.F. was visiting the Bureau d' Economie Théorique et Appliquée (BETA), Université Louis Pasteur, Strasbourg, France. G.F. gratefully acknowledges financial support by the CNRS (Centre National de la Recherche Scientifique), France. All usual caveats apply.

\section{References}

1. Albert R, Barabási A (2002) Statistical mechanics of complex networks. Rev Mod Phys 74:47-97

2. Amaral L, Scala A, Barthélemy M, Stanley H (2000) Classes of small-world networks. Proc Natl Acad Sci 97:11149-11152

3. Baldwin T, Rozenberg G (2004) Britain 'must scrap multiculturalism'. The Times, April 2003

4. Barabási A, Albert R (1999) Emergence of scaling in random networks. Science 286:509-512

5. Bollobás B (1985) Random graphs. Academic, New York

6. Dorogovtsev S, Mendes J (2003) Evolution of networks: fom biological nets to the Internet and WWW. Oxford University Press, Oxford

7. Echenique F, Fryer R (2005) On the measurement of segregation. Working Paper No. 11258, NBER

8. Fagiolo G (1998) Spatial interactions in dynamic decentralized economies: a review. In: Cohendet P, Llerena P, Stahn H, Umbhauer G (eds) The Economics of networks. Interaction and behaviours. Springer, Berlin

9. Fagiolo G, Valente M, Vriend N (2007) Segregation in networks. J Econ Behav Organ 64: 316-336

10. Fershtman M (1997) Cohesive group detection in a social network by the segregation matrix index. Soc Networks 19:193-207

11. Freeman L (1972) Segregation in social networks. Sociol Methods Res 6:411-427

12. Freeman L (1978) On measuring systematic integration. Connections 2:9-12

13. Milgram S (1967) The small world problem. Psychol Today 2:60-67

14. Mitchell J (1978) On freeman's segregation index: an alternative. Connections 2:9-12

15. Newman M (2000) Models of the small world: a review. arXiv:cond-mat/ 0001118v2

16. Newman M (2003) The structure and function of complex networks. SIAM Rev 45:167-256

17. Pancs R, Vriend N (2007) Schelling's spatial proximity model of segregation revisited. J Public Econ 91:1-24

18. Pastos-Satorras R, Vespignani A (2004) Evolution and structure of the Internet. Cambridge University Press, Cambridge

19. Schelling T (1969) Models of segregation. Am Econ Rev 59:488-493

20. Schelling T (1971) Dynamic models of segregation. J Math Sociol 1:143-186

21. Schelling T (1971) On the ecology of micromotives. The Public Interest 25:61-98

22. Schelling T (1978) Micromotives and macrobehavior. W.W. Norton, New York

23. Scott J (2000) Social network analysis: a handbook. Sage, London

24. Wasserman S, Faust K (1994) Social network analysis. methods and applications. Cambridge University Press, Cambridge

25. Watts D (1999) Small worlds. Princeton University Press, Princeton

26. Watts D (2003) Six degrees: the science of a connected age. W.W. Norton, New York

27. Watts D, Strogatz S (1998) Collective dynamics of 'small-world' networks. Nature 393:440-442 